\title{
LA ANTIGÜEDAD DE ASENTAMIENTOS INFORMALES COMO FACTOR DE DIFERENCIACIÓN SOCIOESPACIAL UN CASO DE ESTUDIO EN LA CIUDAD DE MÉXICO
}

\section{AGE OF INFORMAL SETTLEMENTS AS A FACTOR OF SOCIAL AND SPATIAL DIFFERENTIATION A CADE STUDY IN MEXICO CITY}

v. 9, n. 1 [15]

jan/abr (2017)

Dossiê "Villas Miseria, Favelas y Asentamientos: nuevas rutas en Historia Urbana"
Milton Montehano Castillo Intituto Politécnico Nacional mmontejanoc@ipn.mx

\section{Resumen}

Los asentamientos informales han formado parte de los modelos de estructura urbana y diferenciación socioespacial de la ciudad latinoamericana en los últimos treinta años. Sin embargo, el crecimiento, consolidación y heterogeneidad creciente de este tipo de asentamientos los ha convertido no solo en un elemento más de la ciudad, sino en una ciudad en sí. A pesar de los retos que tales ciudades informales presentan en términos de planificación y gestión, no ha habido ningún intento por modelar su estructura y transformación. Por ello, en este artículo se propone la lectura histórica como base para entender la diferenciación socioespacial de la ciudad informal. El caso de estudio es Ciudad Nezahualcóyotl, México; una ciudad de origen informal de más de un millón de habitantes y más de cincuenta años de transformación, lo que permite identificar algunos elementos de diferenciación intraurbana.

\section{Palavras clave}

Asentamiento informal. Historia urbana. Diferenciación socio-espacial. Ciudad de México.

\footnotetext{
Abstract

Informal settlements have been part of the models of urban structure and socio-spatial differentiation of the Latin American city in the last thirty years. However, growth, consolidation and increasing heterogeneity of such settlements make them not just an element of the city but
} 
a city in itself. Despite the challenges that such informal cities present in terms of planning and management, there has been no attempt to model their structure and transformation in time. Therefore, in this article we propose the historical perspective as a base to understand the sociospatial differentiation of the informal city. The case study is Ciudad Nezahualcoyotl, Mexico; a city with an informal origin with more than one million inhabitants and more than fifty years of transformation, where in principle is possible to identify some elements of intraurban differentiation.

\section{Keywords}

Informal settlements. Urban history. Social and spatial differentiation. Mexico city.

\section{Los asentamientos informales y su heterogeneidad socioespacial}

En Latinoamérica, los asentamientos informales aparecieron generalmente como consecuencia de la rápida urbanización e industrialización ocurrida después de la segunda guerra mundial, en parte debido al crecimiento natural y la migración rural-urbana. En adición a este crecimiento, una rápida polarización social se reflejó en el mercado inmobiliario. Debido a bajos salarios y la precaria naturaleza del desarrollo económico de esa área, la vivienda para la clase trabajadora no fue una prioridad. La población de escasos recursos adquirió vivienda en algunos casos subdividiendo mansiones abandonadas por las clases pudientes. La vivienda alternativa de bajo costo apareció, con gente construyendo en terrenos invadidos y posteriormente con la compra de terreno con ayuda de fraccionadores ilegales (RIBBECK, 2002). Estos asentamientos se esparcieron en todas las áreas urbanas. En muchos casos, se localizaron en terrenos que eran inadecuados para la construcción de vivienda debido a riesgos por inundación o pendientes muy pronunciadas donde la construcción fue muy difícil y el abastecimiento de infraestructura muy costoso.

Con el tiempo, la construcción de asentamientos informales se convertió en una opción atractiva aún para las clases medias. Muchos habitantes de clase media en países en desarrollo comenzaron a construir sus propias viviendas en desarrollos no autorizados o invadiendo terrenos. Un aspecto positivo de este reacomodo social ha sido que los niveles de educación de las clases medias les han permitido participar en la formulación de nuevas políticas de vivienda. Por otro lado, los precios de las propiedades continuaron incrementándose, lo que causó subdivisiones posteriores de los lotes y rentas altas. Dentro de estos procesos de urbanización, los asentamientos informales se han hecho también mucho más complejos que hace dos o tres décadas. Los estudios comparativos a nivel global permiten ver que la organización social y espacial de los asentamientos informales es cada vez más y más heterogénea y de ninguna manera, son los grupos de bajos recursos los que habitan ésas áreas. Económicamente el ingreso de los habitantes de asentamientos informales, proviene sobre todo de actividades informales 
dentro o fuera de los asentamientos informales (UN-HABITAT, 2003, pp. 23-31). El impacto económico de estas actividades es tal, que esos negocios informales con frecuencia no se limitan al área, sino que permean toda la ciudad a través de una compleja red que hace muy difusa la distinción entre la economía formal e informal. En los asentamientos más antiguos se observan notables diferencias en las condiciones de vida e infraestructura, en comparación con asentamientos nuevos. Por lo tanto, las autoridades toman más en cuenta los asentamientos jóvenes que los antiguos y más consolidados, aunque se debería poner mucha más atención al mejoramiento y revitalización de asentamientos ya consolidados para evitar que estos se vuelvan a convertir en áreas de condiciones de vivienda inadecuadas (WARD, 2001, pp. 238243; 2015, pp. 373-384).

Después de muchas décadas de la política y las acciones utilizadas para abordar el fenómeno de la urbanización informal, hoy las estrategias para mejorar los medios de vida de los habitantes de estos asentamientos son múltiples: mejoras en la infraestructura, enfoques eco-sistémicos para reducción del riesgo de desastre, integración de los asentamientos a la dimensión social, económica, política y cultural de las ciudades, entre muchas otras estrategias (UN-HABITAT III, 2016, en línea). Espacialmente, la asignación de recursos a las zonas pobres requiere un mapa geográfico de la pobreza. Sin embargo, esta tarea representa un reto debido a que estos asentamientos son raramente documentados en registros o fuentes oficiales. Por otra parte, la trama urbana producida en la ciudad informal no es sencillo y la estructura social en asentamientos irregulares está lejos de ser homogénea dentro de una misma ciudad o incluso dentro de un asentamiento (UN-HABITAT, 2003, pp. 169).

Debido al tamaño que algunos asentamientos alcanzan, un nuevo término para describir estas grandes aglomeraciones urbanas comienzan a ser usados: la ciudad informal, un nuevo tipo de ciudad caracterizado por una lógica contradictoria: "es a la vez efímera pero extensiva, irrelevante pero crítica, visible pero rara vez observada" (WRIGHT, 2005, pp. 79-82). Otros autores definen la ciudad informal como aquella que comprende "los slums de las megaciudades en desarrollo, en donde el sector informal tiene su base, donde los servicios son pobres o inexistentes; donde los residentes son invisibles a los sistemas legales; y donde el odio de las autoridades (hacia estos asentamientos) es común" (UN-HABITAT, 2003, pp. 23).

Sin embargo, el tejido urbano producido en la ciudad informal no es simple, y el mejoramiento de las vidas de los residentes de las ciudades informales no solo consiste en convertir lo informal en formal. Junto con los procesos físicos de urbanización informal, otras fases o sub-procesos se realizan, tales como la consolidación del mercado inmobiliario (el momento en que la vivienda se convierte en mercancía), consolidación legal y consolidación socioeconómica (ZILLMAN, 2000, pp. 193-206). En adición, los cambios que ocurren en los asentamientos pueden observarse de manera lineal o puede ocurrir un "estancamiento". Estos procesos de cambio también pueden ser acelerados debido a una variedad de factores tales como la organización comunitaria, la actitud de las autoridades, las mejoras en el transporte, la 
atracción de inversión privada o pública dentro del área, o el crecimiento de la ciudad, por mencionar solo algunos. Por lo tanto, el tejido urbano resultante consiste en una mezcla funcional y social de áreas semi-consolidadas, colonias donde predomina la vivienda en renta, y asentamientos bien establecidos donde predomina el comercio. La ciudad informal debería ser entonces reconocida como una parte integral de la ciudad con un tejido urbano específico que debería ser considerado por urbanistas y políticos (ZILLMAN, 1998, pp. 26-35).

\section{Modelos de diferenciación socioespacial en los asentamientos informales}

Como objeto de análisis geográfico urbano, los asentamientos informales, han formado parte de los modelos de estructura urbana y diferenciación socioespacial elaborados en los últimos 30 años. En algunos de estos modelos se menciona de manera general la presencia de los asentamientos informales periféricos (FORD, 1996, pp. 437-440) o se hace cierta distinción entre ellos, como por ejemplo: barrios marginales, barrios marginales periféricos, y barrios antiguos ya consolidados (BORSFORF, 2003, pp. 37-49), o barrios marginales centrales, barrios marginales periféricos, o antiguos barrios marginales consolidados (BORSDORF, A.; BÄHR, J. \& JANOSCHKA, M., 2002, pp. 300-310). Como su nombre lo indica, la localización de estos asentamientos en ocasiones es central o periférica, consolidados o antiguos. Sin embargo, el crecimiento, consolidación y heterogeneidad creciente de este tipo de asentamientos los ha convertido no solo en un elemento más de la ciudad latinoamericana, sino en una ciudad en sí con una geografía interna propia, aunque esta diferenciación interior no ha sido plenamente analizada.

En la década de 1960, los asentamientos informales aún eran considerados una especie de amenaza social. En este contexto, existía una necesidad urgente de evaluar el potencial de mejoramiento de los asentamientos en el largo plazo. El resultado fue un marco teórico desarrollado por Charles Stokes alrededor de 1962 (STOKES, 1962, pp. 187-197).

De acuerdo a Stokes, dos variables eran esenciales para determinar el potencial de mejoramiento de los asentamientos informales en el largo plazo. La primera variable era la motivación del "habitante del slum" (slum dweller) con respecto a cambiar social y económicamente, algo definido como escalación. La segunda variable era la capacidad y la habilidad del habitante (por ejemplo educación), para ser integrado en la vida social y económica de la ciudad. La fórmula concreta propuesta por Stokes era una correlación numérica entre la demanda de trabajo y la oferta de mano de obra existente, en un contexto económico de un país en desarrollo.

De acuerdo a Stokes, un grupo de habitantes de un "slum", con la actitud psicológica de "esperanza" (hope) y con las habilidades necesarias para "escalar", podría ser eventualmente integrado a las actividades económicas de una ciudad. Como consecuencia, esos habitantes estarían en posición de mejorar su asentamiento. En el caso contrario, un grupo con la actitud de "desesperanza" (dispair), no estaría en posición de conseguir un trabajo y por lo tanto un 
salario. Sin los medios económicos para mejorar el asentamiento, éste eventualmente se tornaría un ambiente indeseable y una amenaza social.

Alrededor de 1960 John Turner (TURNER, 1972) argumentaba que la escalación social y económica de los habitantes de bajos ingresos solo sería posible si el gobierno dejara de dictar la forma y la función de dichos asentamientos. De acuerdo a Turner, si no se realizaba ninguna intervención pública en el asentamiento, los habitantes tendrían el potencial de mejorar sus condiciones de vivienda por cuenta propia en el largo plazo. Turner también argumentaba que la población de bajos ingresos no solamente era dinámica económicamente, sino también dinámica espacialmente. Turner basaba sus argumentos en el supuesto de que los individuos tienen prioridades diferentes con respecto a la localización de su vivienda:

a) La necesidad de vivir cerca del centro de la ciudad y de oportunidades de trabajo, dándole menos importancia a la calidad del asentamiento.

b) La necesidad de tenencia de la vivienda, y

c) La necesidad de calidad y tenencia de la vivienda, dándole menos importancia a vivir cerca de las oportunidades de trabajo.

Turner asociaba estas tres prioridades con una tipología de habitantes de bajos ingresos: Habitantes de muy bajos ingresos, por ejemplo un recién inmigrante, Habitantes de bajos ingresos, Habitantes de ingresos medios y Habitantes de ingresos medio altos (por ejemplo un inmigrante social y económicamente integrado a la ciudad).

Alrededor de la década de 1960, también se argumentaba que los asentamientos informales de ninguna manera tenían el potencial de mejoramiento, sino que más bien estos eran comunidades explotadas por una fuerza económica muy poderosa. Desde esta perspectiva, las reglas del sistema económico eran reproducidas dentro de los asentamientos informales, pero a una escala pequeña, por ejemplo, renta informal de vivienda, pequeños prestadores de dinero, vendedores de material de desecho informales (MATHÉY, 1988, pp. 43-51). Una metodología para analizar los asentamientos desde este enfoque, consistiría en identificar la correspondencia entre las categorías de la economía formal e informal.

Investigaciones a inicios del siglo mostraban que los asentamientos informales no eran comunidades estáticas, sino dinámicas, y el reconocimiento de esto era esencial para la formulación de intervenciones externas (UN-HABITAT, 2003, pp. 79-95). Para estimar el potencial de mejoramiento de asentamientos informales, por lo menos dos cosas deberían ser tomadas en cuenta; a saber la tenencia de la vivienda y las intervenciones para el mejoramiento que se hayan hecho anteriormente. En este sentido, podrían identificarse tres tipos de asentamientos informales:

a) Asentamientos mejorados a través de la intervención. Corresponde a los asentamientos en donde se ha hecho alguna intervención para mejorar uno o varios aspectos del asentamiento, sin embargo, no han sido objeto de un proyecto integral de mejoramiento. 
Como tales, estas intervenciones han sido el resultado de un esfuerzo que ha tomado tiempo y participación de los habitantes, y probablemente ayuda a crear un sentido amplio de comunidad.

b) Asentamientos Mejorados (upgraded slums). Estos son asentamientos informales que han sido objeto de un programa comprehensivo de mejoramiento. Sin embargo, no hay garantía de que los mejoramientos duren mucho. Posteriormente, las condiciones de mejoramiento pueden servir para atraer más residentes al asentamiento, incrementando la presión en el crecimiento de la vivienda y servicios, para crear una vez más condiciones inadecuadas de vivienda.

c) Asentamientos que carecen de incentivos para mejoramiento. Estos son asentamientos informales en los cuales los residentes solamente aspiran a tener los mínimos estándares de vivienda y por lo tanto no tienen incentivos para llevar a cabo medidas de mejoramiento.

Por otra parte, las condiciones descritas anteriormente han dado lugar a una serie de trabajos para explicar la consolidación de asentamientos informales dentro de una ciudad (HILLIER, et. al., 2000: 61-96) o de mapear de pobreza, incluso frente a la escasez de fuentes oficiales o censos disponibles. Los asentamientos informales se han interpretado entonces como una "textura urbana" con el fin de correlacionar los patrones morfológicos con la pobreza (BARROS Y SOBREIRA, 2005; DA PENHA PACHÊCO ET.AL, 2014, pp. 143-159). No obstante, sigue siendo necesario profundizar en la caracterización de áreas geográficas que relacionen patrones físicos con variables socioeconómicas y sus transformaciones en el tiempo en el ámbito de la informalidad urbana.

Considerando el tipo de vivienda, la tipología es mucho más extensa, de especial interés son los asentamientos localizados ilegalmente en terrenos de propiedad privada. Tales asentamientos aparecen cuando existe una figura importante como un líder político u otros intermediarios para proveer protección. Para estos asentamientos la seguridad de la tenencia de la vivienda es el principal objetivo, con todo el potencial de mejoramiento que la seguridad de la tenencia trae consigo.

\section{El caso de ciudad Nezahualcóyotl, Ciudad de México}

A pesar de su naturaleza informal, los límites entre la urbanización formal e informal se han hecho muy difusos con el tiempo. Durante el proceso de evolución de los asentamientos informales se pueden identificar tres generaciones (RIBBECK, 2002, pp. 72-78). La primera generación fue la de invasores que carecían de toda organización. Pero con el tiempo, y debido a los cada vez más escasos terrenos, una segunda generación de asentamientos informales apareció. Estos incluían subdivisiones ilegales de tierra, pero sin servicios ni diseño urbano. Una tercera generación se caracteriza por la cooperación entre desarrolladores informales y autoridades de planeación, ofreciendo por lo menos un mínimo de terrenos para servicios y equipamiento. Este sería el caso de Ciudad Nezahualcóyotl (Ilamado de manera corta "Neza"), 
originalmente nació como un asentamiento informal, está localizado al oriente del Área Metropolitana de la Ciudad de México. Ciudad Neza ha sido autoconstruida en los últimos cincuenta años y con una superficie de $25 \mathrm{~km}^{2}$ y más de un millón doscientos mil habitantes, es uno de los asentamientos informales más grandes y densamente poblados del mundo. Como la mayoría de los asentamientos, este se ha desarrollado y transformado de manera más bien caótica que sistemática. Algunas etapas "históricas" deben ser consideradas para entender la evolución y la correspondiente diferenciación interna de la ciudad.

La actual Ciudad Nezahualcóyotl, nombrada así en honor a un rey y poeta texcocano, aparece sobre la región del secado lago de Texcoco, que se emplazaba anteriormente en el valle de la actual ciudad de México y donde, desde el siglo 17, se realizaron una serie de intentos de drenaje, que finalmente en el siglo 20 llevaron casi a la desecación del lago.

Junto con el arribo de los primeros habitantes a principios del siglo $\mathrm{XX}$, aparecieron confusiones en relación a la propiedad debido a registros defectuosos, los cuales se agravaron en 1940 dando lugar a una absoluta confusión: la tenencia de la tierra estaba dividida entre el Estado, los ejidos y la propiedad privada y los límites de los lotes estaban indefinidos, de tal manera que los lotes individuales parcialmente pertenecían a varios dueños.

Por este tiempo vivió la capital de México una ola de industrialización que motivó una migración masiva, y a comienzos de la década de 1940 una creciente industrialización permitió la integración de la creciente fuerza de trabajo en las instalaciones de producción de la capital.

Sin embargo, con la crisis agraria desatada en la década de 1950, la migración rural en la capital creció y esto condujo a un rápido crecimiento de la población. Sumado a esto, la industria bajó su necesidad de fuerza de trabajo humana a través de un incremento de inversiones de capital, lo que subió el desempleo en la ciudad e indirectamente se forzó a los migrantes a tomar ocupaciones lejos por debajo de los ingresos mínimos reglamentarios (SCHTEINGART, 1981).

Debido a que el acceso a una vivienda bajo tales condiciones de ingresos era prácticamente imposible, la clase social afectada recurrió a otros métodos, frecuentemente ilegales, para acceder a suelo y a una posible vivienda. A ello se añadió en 1945 una ordenación que prohíbe la construcción de fraccionamientos dentro del Distrito Federal.

Estos dos factores favorecieron en los años 50 una ampliación de la urbanización sobre las fronteras administrativas del Distrito Federal hacia los municipios vecinos y sobre todo sobre los terrenos del desecado lago de Texcoco, cuya confusión de propiedad favoreció dudosas prácticas de venta de terreno.

Para 1958, cuando el número de habitantes que existía en estas tierras ascendió a 12,000, intervino el Estado Federal y forzó a los fraccionadores a construir equipamiento e infraestructura (Agua, Drenaje, Energía y pavimentación), así como a la adaptación de una red de parcelamiento. El principio de parcelamiento introducido fue caracterizado por una estricta ortogonalidad, que aún hoy define la ordenación espacial en Nezahualcóyotl (ver Figura 1). Se 
trata de una superficie que es estructurada por 13 ejes principales de $40 \mathrm{~m}$. de ancho con una distancia correspondiente entre 1000 y 1200 metros. Cuatro de estos ejes corren en el sentido largo y nueve perpendiculares a ellos forman las barreras de los grandes bloques ("supermanzanas").

A la mitad de cada gran manzana se dejaron libres superficies para servicios públicos y mercados y las supermanzanas.se dividieron posteriormente en pequeñas manzanas (de 200 por 30m aproximadamente), dando como resultado una superficie con unas 4500 manzanas. Cada manzana contiene unos 50 pequeños lotes de unos $150 \mathrm{~m}$. y los lotes limitan con la calle por su lado estrecho (TESCHNER, 1991, pp. 24-32).

Estas medidas sin embargo no fueron consideradas por los desarrolladores informales para toda la ciudad. En algunas áreas que ya habían sido ocupadas, los desarrolladores se negaron a donar lotes para la creación de áreas públicas, dando como resultado supermanzanas con variaciones en su forma y en la distribución de sus áreas. Algunas supermanzanas fueron creadas con áreas públicas dispersas o con grandes zonas ortogonales que medían aproximadamente $500 \times 120$ metros. Otras supermanzanas agrupaban sus áreas públicas en una, dos o tres plazas que limitaban con las avenidas (ver Figura 1).

En general las medidas de planeación de 1958 no fueron llevadas a cabo en la realidad, pues la venta de las parcelas continuó a través de los fraccionadores sin asegurar la tenencia, equipamiento e infraestructura.

Debido a que la superficie pertenecía a más de un municipio, la realización de los mejoramientos prescritos de la infraestructura y el acuerdo de su construcción fueron difíciles. Por este motivo se aspiró entonces a una administración propia que pudiera asegurar una toma de decisiones autónoma y en 1963 se logró finalmente su independencia administrativa (ver Figura 1).

La autonomía no llevó sin embargo a los resultados esperados. La construcción de la infraestructura fue realizada por las primeras autoridades locales de manera incompleta, así que a partir de 1970 hizo presencia una fuerte organización de colonos Ilamada "Movimiento Restaurador de Colonos" -MRC-, que trataba de forzar a cada fraccionador a construir la infraestructura prometida. Así, la organización de un paro en los pagos de lotes -hacia los fraccionadores- tuvo como efecto fuertes reacciones para los fraccionadores, que esperaban su dinero. Como reacción a la situación, los fraccionadores informales, con ayuda de la policía local y con apoyo de las autoridades, trataron de desalojar a los colonos que no pagaban, aunque estos intentos terminaron en fuertes choques entre los colonos y la policía (VEGA, 1991, pp. 161-177). Los continuos conflictos terminaron en 1973 a través de un ordenamiento del presidente Luis Echeverría, quien exigió a los colonos a pagar completamente sus lotes. El dinero debía pagarse no a los fraccionadores sino al Estado, por lo que recibieron los colonos $15 \%$ de rebaja. Del total del dinero, el Estado pagaría el $40 \%$ a los fraccionadores y del restante $60 \%$ 
del dinero, se tendría que pagar entre la infraestructura faltante y los fraccionadores debían regresar el resto de los lotes al Estado (SCHTEINGART, op. cit).

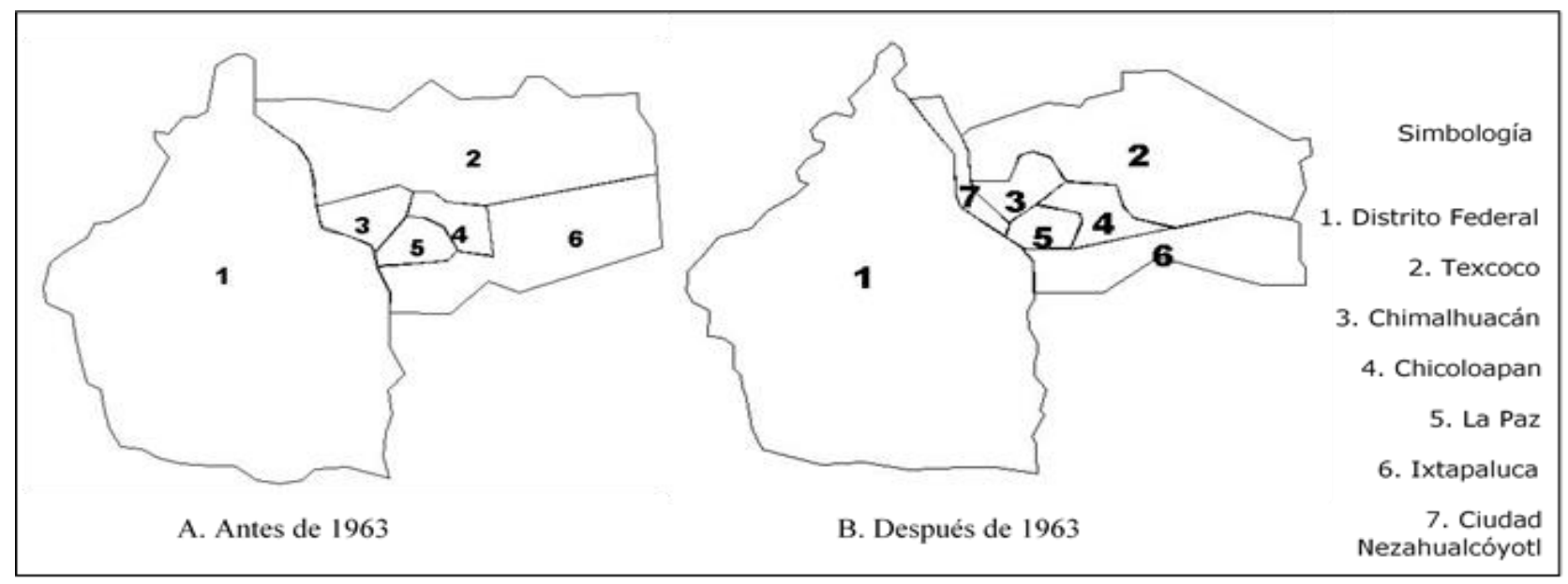

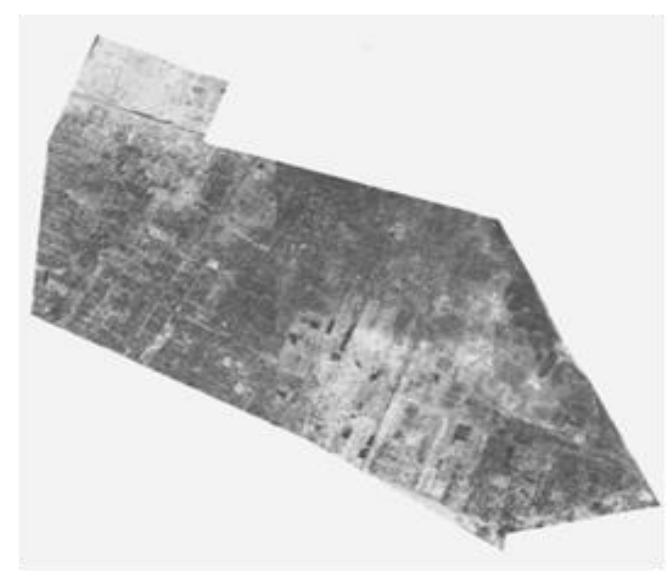

Ciudad Nezahualcóyotl, 1967

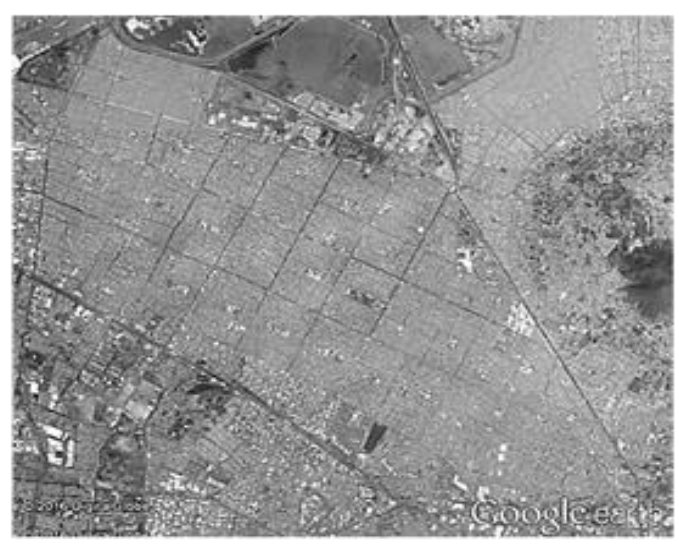

Ciudad Nezahualcóyotl, 2016

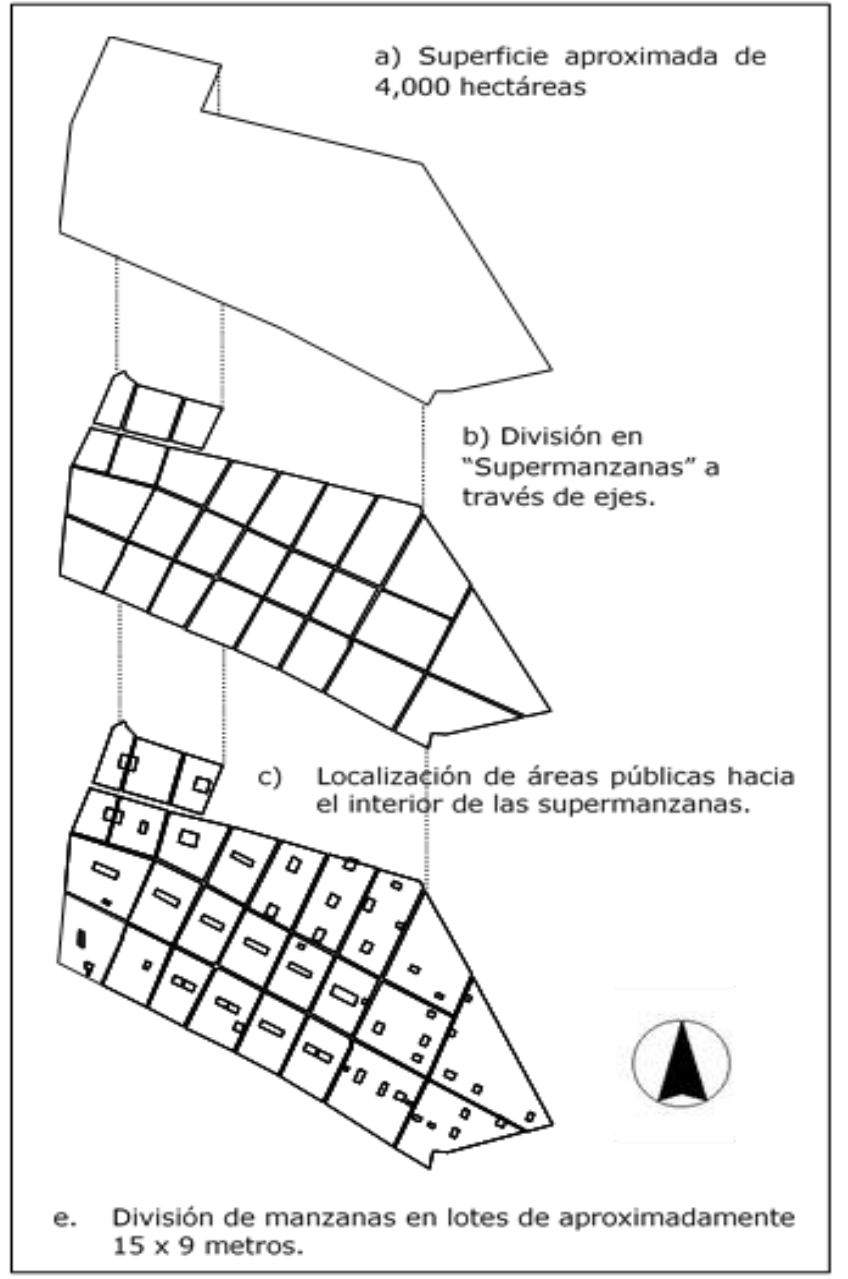

Figura 1. Creación del municipio de Ciudad Nezahualcóyotl y lotificación

Fuentes: Fotografía aérea tomada de: Aerofotogrametría Internacional Struck, S. A. vuelo 1967 (foto superior) y Google Earth, 2016 (foto inferior). Dibujos del autor. 
Del acuerdo de 1973 se pudieron invertir 150 millones de dólares en agua y drenaje, electricidad y pavimentación (TESCHNER, 1991, pp. 24-32). Con la construcción de la infraestructura comenzó al mismo tiempo el proceso de legalización, el cual concluyó en 1990, cuando el Gobernador del Estado entregó los últimos 3000 títulos. Con la conclusión de esta fase de legalización, oficialmente 160000 lotes estaban en manos de los colonos.

A través de las iniciativas de los propios habitantes, especialmente a través de las acciones del MRC, se disparó una ola de iniciativas de consolidación, cuyos resultados en los años posteriores todavía se podían reconocer: en 1980 más del 90\% de las viviendas están conectadas a la red de agua y drenaje y $96 \%$ conectadas a la luz eléctrica (INEGI, 1980, 1990). En 1990 la infraestructura todavía se amplió, así que casi el 100\% de las viviendas tenían acceso a agua y electricidad.

Haciendo un balance de los resultados, los logros alcanzados por el movimiento de colonos se debe considerar sin embargo como relativo. Aun cuando entre las décadas de 1970 a 1990 se logró que casi todas las viviendas tuvieran acceso a energía, agua y drenaje, la capacidad y características de la red ya en ese tiempo no era suficiente y con aumento de la población ha habido cada vez más déficits que apuntar, lo que ha tenido consecuencias no sólo para los pobladores sino también para el medio ambiente.

Los terrenos sobre los que descansa Ciudad Nezahualcóyotl son completamente planos. Por este motivo y bajo las condiciones climáticas, se hace necesario un equipamiento especial para drenaje y abastecimiento de agua. Las bombas que drenan el agua de lluvia y de drenaje están cuantitativa y cualitativamente en un estado, en el que en el tiempo de lluvias no pueden garantizar que la ciudad no se inunde. A ello se añade además el desgaste y hundimiento en cada vez capas más profundas de suelo a través de la construcción de pozos profundos, para combatir el déficit de abastecimiento de agua.

No sólo el abastecimiento de la infraestructura sino también la red de calles que une la ciudad con los municipios colindantes, no corresponde con los requerimientos de tráfico actuales, en lo que toca los aspectos cuantitativos. Las pocas calles principales se saturan a través del creciente tráfico. El tráfico crece en las calles internas, donde diariamente ocurren congestionamientos y accidentes.

Por otra parte, se reconoce que la ordenación espacial de la ciudad, en especial la cantidad, tamaño y uso de espacios públicos dentro de las supermanzanas es limitada actualmente a las necesidades de los habitantes. Especialmente problemático parece la escasez y práctica inexistencia de instalaciones del tiempo libre para jóvenes. Con ello, la falta de instalaciones deportivas y culturales conduce a que el uso del tiempo libre que se lleva a cabo en las calles.

No menos importante es la situación económica de los habitantes. Aunque en Neza ya se ha desarrollado una economía local, esta oferta no representa una fuente de trabajo segura 
y suficiente, lo que conduce a una elevada integración de negocios sin autorización e industria ilegal en los hogares.

Al parecer, según la escala y naturaleza de los problemas, ocurren mecanismos de autoorganización, los cuales desaparecen lentamente cuando el asentamiento alcanza la dimensión de una ciudad y las posibilidades de solución de sus problemas se hacen más complejas.

Dentro de la problemática expuesta y dada la existencia de documentos, se puede tener un panorama general sobre las condiciones de la ciudad, pero es difícil poder identificar diferencias intraurbanas, pues el proceso de construcción y transformación de la vivienda ha sido básicamente el mismo.

Desde su fundación hasta finales de los años 60, Ciudad Neza no presentó diferenciaciones internas, pues como se observó, históricamente, las primeras décadas son de consolidación hasta convertirse en municipio. Una primera diferenciación interna para Ciudad Nezahualcóyotl puede observarse en la etapa de los comienzos del proceso de urbanización en la década de 1970. Durante esa etapa, la diferenciación no era mucho el resultado o la expresión de necesidades individuales, sino una expresión de necesidades colectivas; necesidades que no eran sentidas solo por un grupo pequeño de habitantes, sino por la población entera. Tales necesidades eran viviendas con acceso a infraestructura, tenencia legal, construcción de equipamiento, estos eran algunos de los parámetros de consolidación en esa década.

\section{Antiguedad y diferenciación socioespacial en Ciudad Neza}

Cuando el asentamiento crece, las características sociales y físicas se hacen cada vez más diversas, así como los parámetros para capturar esta diversidad. Estos parámetros incluyen una mezcla de viviendas consolidadas con viviendas no-consolidadas, familias con necesidades diferentes, así como la función comercial de cada colonia. Esta última aun con más elementos de análisis si se toma en cuenta que, con el tiempo, la diferenciación funcional no solo es relativa a las necesidades de la población local, sino también es relativa a la población de alrededor. En el caso en cuestión, un factor determinante ha sido su colindancia con la ciudad de México y con los municipios de Chimalhuacan y los Reyes. En el primer caso, la ubicación de Neza en relación a la ciudad de México, ha influido desde sus inicios, hasta la actualidad, lo cual se muestra a lo largo de los datos de investigación presentados.

Con base en la información registrada para Ciudad Nezahualcóyotl, la diferenciación social y espacial de la ciudad se determina y presenta a continuación. De acuerdo a las características del municipio y según los datos obtenidos y buscando una mejor interpretación de las diferenciaciones espaciales, se propone una lectura por zonas en función de su antigüedad, las cuales son descritos a continuación (ver Figura 2). 


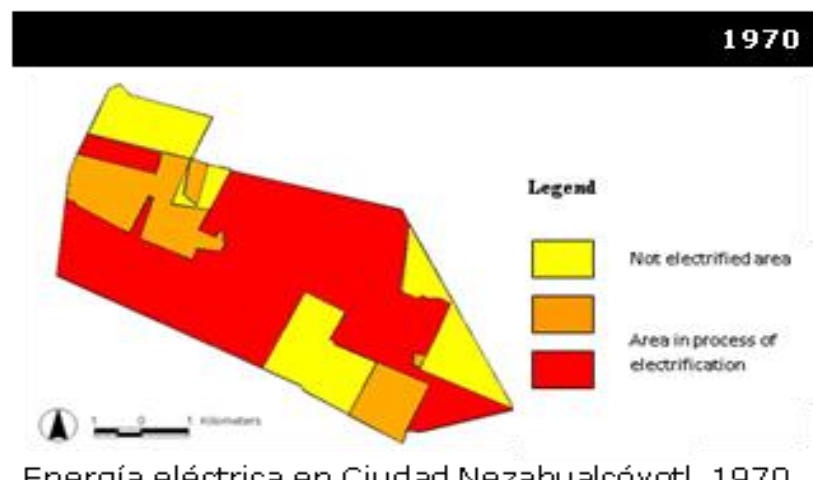

Energía eléctrica en Ciudad Nezahualcóyotl, 1970

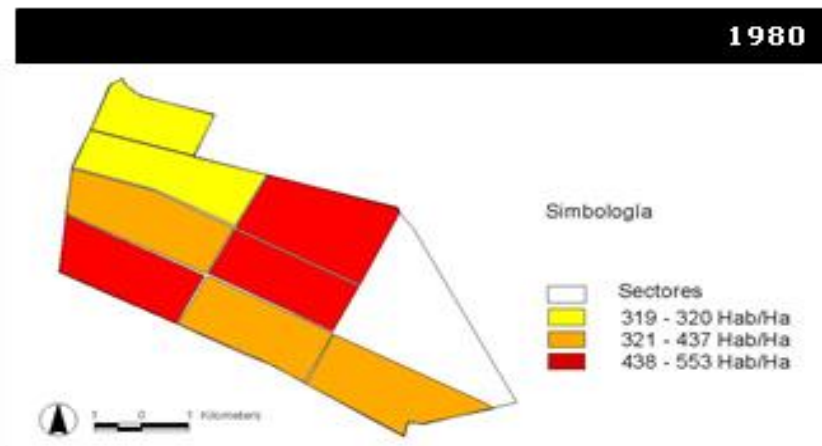

Densidad de población en in Ciudad Nezahualcóyotl, 1983

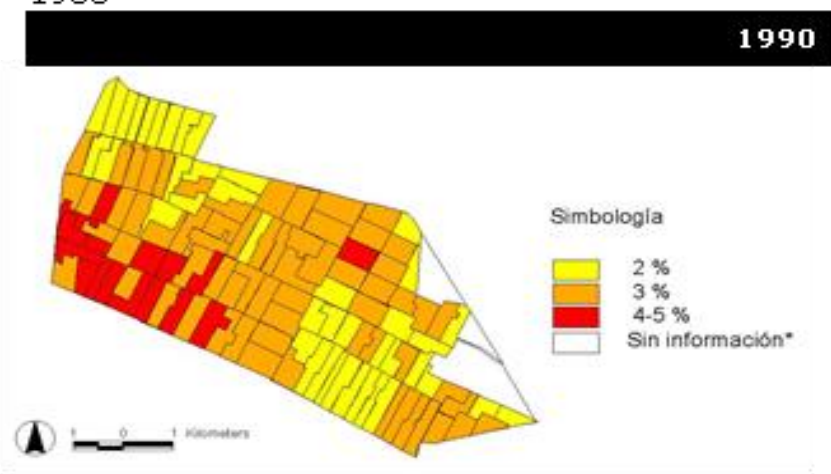

Población mayor de 65 años y más en Ciudad Nezahualcóyotl, 1990 (Porcentajes)

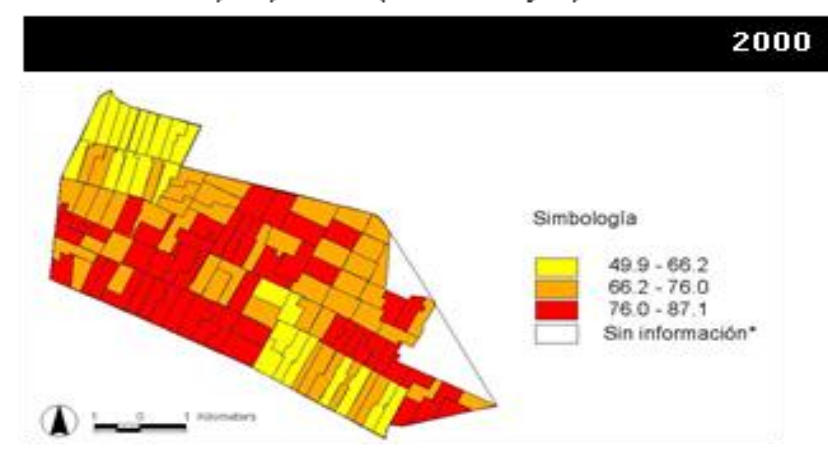

Viviendas con techo de concreto en Ciudad Nezahualcóyotl, 2000 (Porcentajes)

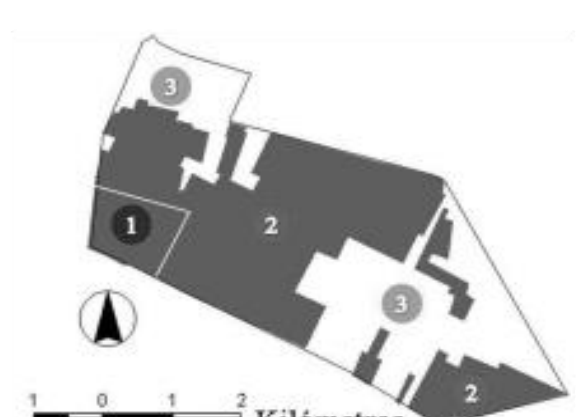

Kilómetros

\section{SIMBOLOGÍA}

\section{1. Área de asentamientos}

tempranos. Ocupada en la década de 1940 aproximadamente.

2. Área de asentamientos antiguos. Ocupada hasta 1967 aproximadamente.
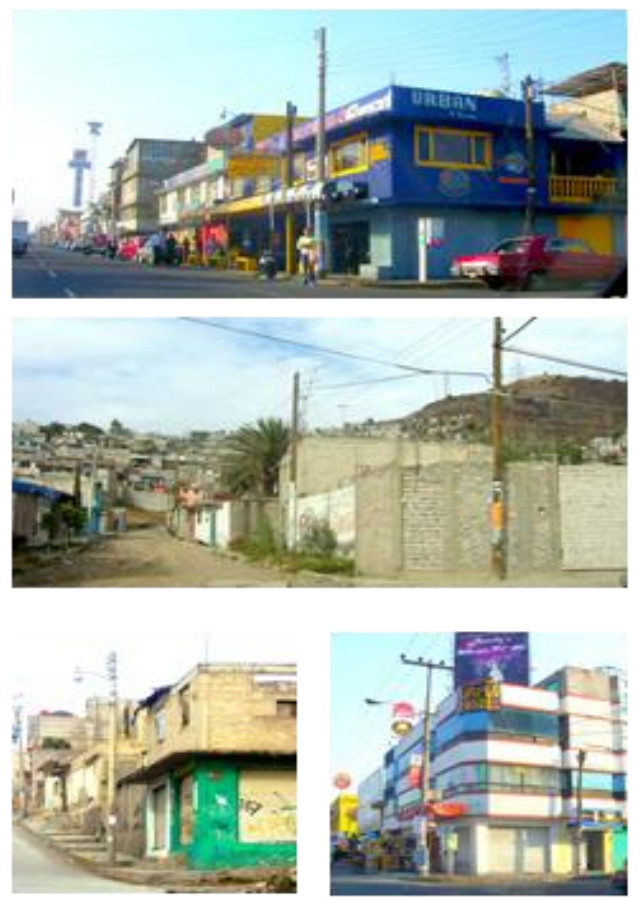

Diferencias en el grado de consolidación y comercialización en Ciudad Nezahualcóyot

Figura 2 - Ciudad Nezahualcóyotl. Viviendas predominantes según edad de las manzanas Fuentes: INEGI, Censos de Población y Vivienda 1990 y 2000. Dibujos y fotos del autor. 


\section{a. Area de asentamientos tempranos (ocupada alrededor de 1940)}

Esta área se ubica en el sur-poniente del municipio, colinda por el poniente y el sur con la ciudad de México y pertenece a los asentamientos tempranos de ciudad Neza.

Como área de Asentamientos Tempranos, ésta se desarrolló antes de que existieran restricciones oficiales sobre el tamaño de los lotes, calles o espacios públicos. Por esta razón, esta área tiene una estructura espacial irregular: no hay una jerarquía clara de calles; las calles no son paralelas al resto de la ciudad y el equipamiento no está ni clara ni equitativamente distribuido. La irregularidad espacial ha sido tal vez uno de los factores que han obstaculizado una integración funcional con el resto de la ciudad, a pesar de su proximidad directa.

En cuanto a su población, muchos de los "consolidadores originales" han regresado o han emigrado a sus Estados de origen. La población que queda está compuesta principalmente por familias jóvenes, la mayoría de ellas son inquilinos e inmigrantes recientes. El nivel de educación e ingreso de los nuevos habitantes es bajo, comparado con el resto de la ciudad. No se ven señales de una "gentrificación" o inmigración de grupos con ingresos más altos, sino un fenómeno de "sustitución de estratos".

Por otro lado, el grado de consolidación física en esta área es alto (más de la mitad de los edificios son de dos niveles o más). Asimismo, esta área contiene los porcentajes más altos de la ciudad de viviendas en renta (más de un tercio de las viviendas es rentada) y considerando el alto número de viviendas en venta y la baja demanda de ellas, el mercado inmobiliario aquí, puede considerarse más bien como inactivo.

Junto con los problemas de infraestructura, poca atención a la revitalización de las viviendas existentes por el lado de los -pocos- dueños, es evidente, así que la imagen general es de deterioro y decremento de la calidad de los edificios, lo que en cambio, favorece aún más la salida de los "consolidadores".

Funcionalmente, el tipo de comercio que se desarrolló en esta área es relativamente tradicional y especializado: existen corredores comerciales de ropa, autopartes y un mercado de carne, cuya mercancía es introducida a la ciudad a través de vehículos de carga que se estacionan en la periferia de Ciudad Nezahualcóyotl, a lo largo del circuito periférico. La existencia de uno de los tianguis más antiguos y el comercio informal que se ha incrementado rápidamente han favorecido el deterioro de la imagen de esta zona.

A pesar de todos los problemas, debido a su localización, ésta área debería ser quizá la que mayor potencial de mejoramiento tiene. Si la mayoría de los dueños no viven ya ahí, una conversión del uso de suelo o el reemplazo de edificios podría ser una opción para el mejoramiento, o una política de inquilinato que beneficie tanto como sea posible a los dueños, a los inquilinos y al interés público de igual manera. Siempre y cuando se planifique una zona de abasto que sustituya esta área.

\section{b. Area de asentamientos antigüos (ocupada alrededor de 1967)}


La determinación de esta área presenta tres partes que son prácticamente centrales al municipio. La más grande se presenta precisamente al centro del municipio y se extiende hacia el lado nororiente, en donde colinda con la vía tapo en una zona federal del ex lago de Texcoco y en la parte poniente con el municipio de Chimalhuacán. Se complementa esta área con una sección ubicada al norponiente del municipio y colindando con el Distrito Federal.

Esta área fue ocupada alrededor de 1960 y puede considerarse como un área de asentamientos antiguos. La estructura espacial es regular y los espacios para equipamiento están homogéneamente distribuidos. Esta área es la más extensa de todas y gracias a su alta densidad y al hecho de que la mayoría de las colonias fueron incluidas en el programa de legalización iniciado en 1973, el proceso de consolidación urbana de ésta área fue relativamente rápido. En 1970 el área completa fue incluida en la construcción de la red de drenaje de la ciudad de Neza y por esos años el área ya estaba abastecida de energía eléctrica. Diez años más tarde, el número de calles pavimentadas había excedido por mucho el de las áreas más jóvenes.

Los grupos de edad de la población son homogéneos: alrededor del 5\% de la población tienen 65 años o más y aproximadamente $30 \%$ de la población tiene menos de 12 años ( $26 \%$ y $33 \%$ en las otras áreas). Dos tercios de las viviendas tienen agua potable dentro del predio y más de tres cuartos de las viviendas tienen techo de concreto. Aproximadamente 1 de 10 viviendas es "cuarto redondo" (en las áreas jóvenes la proporción es de 1 por cada 5). Aproximadamente la mitad del total de las viviendas han alcanzado un nivel de saturación (más de tres niveles) y el resto de los edificios están en proceso de consolidación. En esta área no se planearon edificios para propósitos administrativos como el Ayuntamiento, y los corredores comerciales existen pero el comercio no es tan intenso como en las otras áreas.

Una segunda área se ubica en la parte central norte del ayuntamiento. Limita al norte con un área federal que ha sido utilizada como tiradero a cielo abierto y la avenida conocida como vía tapo, que fue concebida para desahogar la salida de autobuses foráneos al oriente de la ciudad de México. Tiene una estructura espacial regular con una clara jerarquía de calles y pocos espacios libres para equipamiento. Esta zona aloja el Ayuntamiento de la ciudad: un edificio cuya localización, - a pesar de ser una ubicación prácticamente periférica-, se ha convertido en uno de los lugares con la mayor actividad de la ciudad. Espacialmente y funcionalmente, el Ayuntamiento ha influenciado sin duda el uso del suelo y el consecuente arreglo espacial del área. Cerca del Ayuntamiento se ha desarrollado diverso tipo de servicios como bancos, mueblerías, restaurantes, tiendas para materiales de oficina y servicios de internet entre otros. Los centros espontáneos han aparecido no solo en frente del Ayuntamiento, sino también a sus espaldas: centros comerciales lineales que se van uniendo a través de los centros de las supermanzanas en una línea imaginaria.

En ésta área el nivel de educación, ingreso e infraestructura son relativamente más altos que en otras zonas. La existencia de uso de suelo comercial parece excluir otros usos como la renta de viviendas y en esta área el porcentaje de viviendas en renta es muy bajo. Al mismo 
tiempo, ésta área parece ser preferida por familias que llegan a Nezahualcóyotl, o familias que han vivido en otras colonias antes. Las razones para escoger ésta área como lugar de residencia pueden ser múltiples, pero un factor muy importante es la existencia del Ayuntamiento, que puede tener el efecto de un atractivo, no solo para actividades comerciales, sino también como un elemento de identidad.

En los útimos años años se ha incrementado el valor de esta área debido a una fuerte inversión tanto privada como pública. A nivel federal y estatal se desarrolló la construcción del circuito mexiquense, que es un libramiento de oriente a poniente, comunicando el estado de Puebla con Querétaro a través del Estado de México sin pasar por el Distrito Federal. La llamada vía tapo juega ahora un rol importante al ser la alternativa por dos accesos directos al circuito mexiquense. Así mismo los terrenos que fueron tiradero a cielo abierto, han sido utilizados en nuevas edificaciones que dan valor y una nueva imagen a esta área. Anexo al hospital público y Centro de Rehabilitación Social (CERESO), que existían ya desde hace más de diez años, se han edificado un Centro de Rehabilitación Infantil Teletón (CRIT), un centro comercial tipo "mall", oficinas del Poder Judicial Federal, una unidad de la Universidad Autónoma del Estado de México y un hospital privado, además de un centro deportivo.

Dichas construcciones han generado una nueva zona comercial de intenso uso, pues la población ha tomado como un atractivo importante el centro comercial. Además de tiendas de todo tipo, cuenta con restaurantes, cinemas y espacios de diversión infantil y juvenil. Esto ha colaborado positivamente no solo a nivel económico, sino también a nivel social y de imagen urbana. Con esto se espera en corto tiempo un alto grado de consolidación y arraigo de la población en esta área.

Una tercera área con la misma antigüedad se ubica en la parte central sur del municipio, colindando con el Distrito federal. La conexión entre ésta área y el Distrito Federal está desarrollada de mejor manera que las otras áreas. La avenida que atraviesa por la mitad de la región tiene la sección más grande de todas las avenidas y estas conectan a Ciudad Nezahualcóyotl con las arterias principales que conducen al Distrito Federal. La accesibilidad aquí se incrementó aún más gracias a la construcción de una estación del metro férreo en su línea " $A$ ", justamente en el cruce entre el corredor principal y la carretera México-Puebla. Si se llega a Ciudad Nezahualcóyotl en transporte público, se toma sólo unos minutos caminar desde la estación de metro a la "puerta" de Ciudad Nezahualcóyotl. Un largo corredor comercial caracteriza esta zona, donde se localiza toda clase de comercio: desde el comercio especializado y tradicional -como partes para máquinas de coser-, hasta cadenas de comida rápida y tiendas de materiales para la construcción. Desafortunadamente también se tienen los problemas del comercio informal, que se incrementa incontroladamente. Algunas calles son usadas para los tradicionales tianguis, pero otras más han permanecido con puestos informales y obstaculizan la circulación, tanto peatonal como vehicular. Generando con ello un marcado deterioro en la imagen urbana. Este corredor era también el único, que contenía algunos elementos para la 
orientación dentro de la ciudad, como la "puerta de la ciudad" (un monumento que simbolizaba la entrada a la ciudad), Desafortunadamente fue demolido recientemente, rompiendo con ello un factor simbólico, que aunque no era estéticamente de los más atractivos, se constituía en un elemento para identificar la entrada a Ciudad Nezahualcóyotl.

El número de viviendas en venta es relativamente bajo así, puede concluirse que la movilidad de la población aquí es más baja que en el área anterior. Los habitantes con el ingreso más alto y mayor nivel de educación también se encuentran dentro de esta demarcación. El nivel de consolidación en esta área es alto también y prácticamente todas las viviendas tienen agua potable dentro del predio. El porcentaje de viviendas rentadas en ésta área es bajo.

Una buena localización y una rápida comunicación con el Distrito Federal parecen ser los factores que retienen a la población en esta área. Sin embargo, en la opinión de los habitantes, los problemas en esta área no difieren mucho de las colonias más jóvenes y menos consolidadas: inseguridad pública, un servicio público deficiente y problemas de infraestructura relacionados a la falta de mantenimiento del drenaje existente y la red de agua potable. A pesar de todo, en tanto que la accesibilidad a ésta área no sea modificada, se puede esperar que el carácter funcional y socioeconómico de este lugar permanezcan.

\section{C. Área de asentamientos jóvenes (ocupada hacia 1980)}

Esta área está compuesta por dos sectres, el primera se ubica en la parte norponiente del municipio. Colinda al poniente con el Distrito Federal y al norte con el Municipio de Ecatepec. Esta zona está particularmente aislada tanto de la misma ciudad de Nezahualcóyotl, como del Distrito Federal; porque el área limita con el resto del lago de Texcoco hacia el norte y limita con el anillo periférico hacia el oeste, el cual fue construido a una altura varios metros sobre el nivel de las colonias. Estas condiciones han convertido a ésta en un área prácticamente sitiada, con comunicación únicamente hacia el lado sur, con la vía Tapo y como la zona más alejada de la zona del ayuntamiento.

Por el mismo aislamiento debido a su ubicación, esta es el área que más atención requiere de todo el municipio. Tiene una estructura espacial regular con espacio para equipamiento bien distribuido. Fue ocupada alrededor de 1980 pero desde entonces las condiciones de vida no han mejorado mucho. En la última década se construyó la línea B del metro férreo y se desarrollaron centros comerciales y se mejoró la vía conocida como avenida central. Esto ha permitido en los últimos cinco años una mejoría acelerada, debido principalmente al comercio y su comunicación con el municipio de Ecatepec.

Debido a la relativa baja densidad, la velocidad de consolidación urbana (Abastecimiento de agua potable, energía, construcción de drenaje), fue muy lenta y el área no se desarrolló económicamente, tal vez como consecuencia del mismo aislamiento espacial. En términos absolutos, la comercialización se incrementó en un lapso de 20 años, pero en términos relativos, 
el área era la menos comercializada de Ciudad de Nezahualcóyotl. Actualmente se ha consolidado, precisamente el desarrollo de comercio formal, pero también ha crecido el informal.

Alrededor del año 2000 la mitad de las viviendas todavía no tenían agua entubada en el predio y casi un tercio de las viviendas tenía techo con materiales diferentes al concreto (los techos eran generalmente de lámina). Morfológicamente esto se refleja en la proporción de "viviendas en consolidación" y la baja proporción de viviendas consolidadas.

Las características de las viviendas se reflejan directamente en la población también. Casi un quinto de la población vive en "cuartos redondos", lo que representa un alto nivel de hacinamiento, pues esta área está compuesta sobre todo de población joven con más alto porcentaje de población infantil, por ejemplo; más de un tercio de la población tienen menos de 12 años. Este panorama se empeora si se toma en cuenta que ésta es un área propensa a inundaciones. Una inundación periódica y casi predecible ocurre en cada temporada de lluvias. Otro de los problemas graves es la inseguridad. Por su lejanía con el ayuntamiento, es esta área hay un alto índice delictivo. Sumado a un tianguis de autos usados.

La singular problemática de ésta área había sido ya conocida desde la década de 1960. En ese tiempo, la restauración del lago de Texcoco había sido planeada, así como la construcción de caminos para comunicar el área con los alrededores. El plan no se llevó a cabo por falta de fondos. La relocalización de la población era parte del plan. Hoy en día, prácticamente un plan comprehensivo, integral de mejoramiento sería la respuesta mínima para tratar de mejorar las calidad de vida en ésta zona.

El segundo sector con la misma antigüedad se ubica en la parte surponiente del municipio, colinda al sur con el Distrito Federal y con el municipio de Los Reyes. Igualmente esta área fue ocupada alrededor de 1980 . Tiene una estructura espacial regular y la distribución espacial del equipamiento es diferente de las otras áreas en tamaño, número y forma geométrica de las áreas públicas. Debido a su relativa baja densidad, la consolidación urbana, como en el área anterior fue lenta. Las cualidades sociales y espaciales de esta área están mezcladas pero en promedio los indicadores de consolidación física de las viviendas son predominantemente bajos: en algunas partes de ésta área la mitad de las viviendas no tienen agua potable dentro del predio y el porcentaje de cuartos redondos alcanza casi un quinto del total. La estructura de la población tampoco es homogénea pero está compuesta por familias predominantemente jóvenes.

Los corredores comerciales se han desarrollado solo en unas pocas manzanas del área. A pesar del bajo nivel de consolidación, a diferencia del área previa, ésta área posee ciertas ventajas dada su localización, la cual puede ser utilizada para elevar la consolidación.

Una de las avenidas que rodean el área es la avenida Texcoco, la cual define el límite administrativo entre Ciudad Nezahualcóyotl y la ciudad de México. A lo largo de esta avenida, el comercio especializado se ha desarrollado en forma de venta de autopartes y materiales de 
construcción. A lo largo de esta avenida también se han construido gasolineras y diversos servicios en los últimos años.

Otra ventaja es que el porcentaje de viviendas rentadas no es tan alto como en el oeste, un hecho que pudiera ser muy positivo para realizar proyectos de mejoramiento porque la mayoría de los dueños todavía viven ahí. Finalmente, debe enfatizarse que ésta área está rodeado de dos de las áreas mejor consolidadas, pero hasta ahora no existe algún proyecto que mejore la conectividad entre estas tres áreas.

\section{Conclusiones}

La edad de los asentamientos informales determina algunos aspectos de diferenciación pero támbién existen otros factores que intervienen en este proceso. Por una parte, la antigüedad influye en la dotación de servicios básicos como energía, pavimentación y equipamiento, puesto que estos servicios responden o se deciden para áreas con mayor densidad de manera prioritaria. La edad del asentamiento tendrá influencia también no solo en los cambios en la vivienda sino también en el uso de suelo del edificio. El edificio entonces se puede convertir en un edificio comercial vertical, un apartamento de renta, a puede permanecer con uso habitacional con la correspondiente tipología morfológica.

Por otro lado, circunstancias no relacionadas con la antigüedad determinan una consolidación más rápida y sus correspondientes efectos en la diferenciación sociespacial, un ejemplo seria la inserción de equipamiento de mayor imporantacia, como el ayuntamiento, o el la cercanía a la red de transporte masivo metro.

Finalmente, cada asentamiento informal tiene su propia historia y condiciones locales muy específicas. Las condiciones naturales y topográficas sin duda alguna tienen influencia en la forma física de los asentamientos. Un asentamiento informal en un cerro o en la costa, se desarrollará en condiciones formales y constructivas muy diferentes de aquellos ubicados en un terreno plano. En conjunto, con los factores mencionados, la localización del asentamiento dentro de la ciudad y las medidas de planeación inducidas localmente tendrán una influencia directa en la forma y velocidad en la que un asentamiento se transforma.

\section{Agradecimientos}

El autor agradece al Instituto Politécnico Nacional (IPN), Secretaría de Investigación y Posgrado (SIP), por el apoyo financiero otorgado para la realización de este trabajo a través del Proyecto SIP-IPN 20131286, así como a los invaluables comentarios y sugerencias realizadas por el Dr. Gerardo Torres Zárate a este texto.

\section{Referencias}

BARROS, JOANA AND FABIANO SOBREIRA. City of Slums: self-organisation across scales. En: CASA Working Paper Series, 55. Centre for Advanced Spatial Analysis, University College 
London, June 2002, disponible linea en: http://www.casa.ucl.ac.uk/working_papers/paper55.pdf.

BORSDORF, A. Cómo modelar el desarrollo y la dinámica de la ciudad latinoamericana. En: EURE. Revista Latinoamericana de Estudios Urbano Regionales, 2003, Vol. 29, No 86, p. 37-49.

BORSDORF, A.; BÄHR, J. \& JANOSCHKA, M. Die Dynamik stadtstrukturellen Wandels in Lateinamerika. En: Geographica Helvetica, 2002, Vol. 4, No 57, p. 300-310.

DA PENHA PACHÊCO ET AL. Sensoriamento remoto de alta resolução espacial na caracterização de assentamentos informais. En: Revista de Geografía Norte Grande, 57: 143-159 (2014)

FORD, L. R. A New and Improved Model of Latin American City Structure. En: Geographical Review 86 (3): 437-440. 1996.

HILLIER, BILL MARGARITA GREENE AND JAKE DESYLLAS. Self-generated Neighbourhoods: the role of urban form in the consolidation of informal settlements". En URBAN DESIGN International 5: 61-96. Stockholm Press, 2000.

INEGI. Censos de Población 1980, 1990. México.

MATHÉY, KOSTA. Selbsthilfe mit Hand und - Kopf! Positionen und Stationen in einer wohnungspolitischen Debatte. En: Trialog, Zeitschrift für das Planen und Bauen in der Dritten Welt. 3. Quartal, 1988. 18: 43-51.

RIBBECK, ECKHART. Die informelle Moderne. Spontanes Bauen in Mexiko Stadt. Awf-verlag. 2002.

SCHTEINGART, M. El proceso de formación y consolidación de un asentamiento popular en México: El caso de Ciudad Nezahualcóyotl. En: Revista Interamericana de Planificación, 15, Nr. 57, 1981, S. $100-114$.

STOKES J. CHARLES. A Theory of Slums. En Land Economics, a quarterly journal of Planning, Housing \& Public Utilities, Volume XXXVIII, Number 3, 187-197, University of Wisconsin, August 1962.

TESCHNER K. Nezahualcóyotl und Chalco, Irreguläre "Großstädte am Rande der Stadt Mexico. En: Trialog 31, 4 Quartal 1991, S. 24-32.

(c) Urbana: Rev. Eletrônica Cent. Interdiscip. Estud. Cid Campinas (SP) v.9, n.1 [15] p.204-223 ISSN 1982-0569 
TURNER, JOHN F. AND ROBERT FICHTER, (eds), Freedom to Build. Dweller Control of the Housing Process. MacMillan. London/New York. 1972.

UN-HABITAT III. NEW URBAN AGENDA. Draft outcome document for adoption in Quito, October 2016. 10 September $2016 . \quad$ Disponible en línea: https://www2. habitat3.org/bitcache/97ced11dcecef85d41f74043195e5472836f6291?vid=5888 97\&disposition=inline\&op=view

UN-HABITAT. The challenge of slums: global report on human settlements, United Nations Settlements Programme. UN-HABITAT/Earthscan, London, 2003.

VEGA, ANA L. Proceso de poblamiento en la zona oriente de la Ciudad de México. El caso de Ciudad Nezahualcóyotl. En: Espacio y Vivienda en la Ciudad de México, Schteingart (coord.), El Colegio de México/Asamblea de Representantes del Distrito Federal, 1991. S. 161-177.

WARD, PETER. Housing rehab for consolidated informal settlements: A new policy agenda for 2016 UN-Habitat III. En: Habitat International, Vol. 50, December 2015, pp. 373-384.

WARD, PETER. Mexiko Stadt in Beckel, L. Megacities. Ein Beitrag der Europäischen Raumfahrtagentur zum besseren Verständnis einer globaler Herausforderung. GEOSPACE Verlag. Salzburg, 2001. Pp. 238-243.

WRIGHT, GWENDOLYN. Informal Cities, Multiple Realities. In Alfredo Brillembourg, Kristin Feireiss and Hubert Klumpner, editors, The Informal City, Prestel, Munich, 2005, Pp. 79-82.

ZILLMAN, KERSTIN. Rethinking the compact city: Informal Urban Development in Caracas, in Mike Jenks: Compact Cities: sustainable urban forms for developing countries. London. 2000. P. 193-206.

ZILLMAN, KERSTIN. Consolidation and densification of informal squatter settlements in Caracas, Venezuela. Trialog, A Journal for Planning and Building in the Third World. 57: 26-35, 1998. 